\title{
A Perceptive Method for Arabic Word Segmentation using Bounding Boxes by Morphological Dilation
}

\author{
Firoj Parwej, Ph.D \\ Department of Computer Science \\ Jazan University, Jazan, Kingdom of Saudi Arabia (KSA)
}

\begin{abstract}
The character recognition comes under applications of image processing. The character samples are stored in a suitable image format in digital form. Each sample in the image form is properly preprocessed, segmented and the required features defining writer invariants are obtained. The word segmentation is one of the major components in document image analysis. It provides crucial information for skew correction, zone segmentation, and character recognition. The word segmentation is an operation that seeks to decompose an image of a sequence of word into sub images of individual symbols. Its decision, that a pattern isolated from the image is that of a word, can be right or wrong. It is wrong sufficiently often to make a major contribution to the error rate of the system. In this paper we introduced Arabic word segmentation for document images are presented. We are using the bounding box regions to enclose the characters of the Arabic words and then the resulting letter spaces are progressively filled to merge the character bounding boxes to get the Arabic word bounding boxes. The proposed technique is completely avoiding the line segmentation process which normally precedes word segmentation in stuffy methods. We have tested appropriate method on documents of Arabic scripts and theirs have obtained encouraging results from proposed technique.
\end{abstract}

\section{Keywords}

Arabic Text Line Segmentation, Connected Component Labeling, Morphological Dilation, Arabic Scripts, Document Analysis, Preprocessing.

\section{INTRODUCTION}

The Word segmentation is one of the major components in document image analysis. Although the word [1] line segmentation for machine [2] printed or hand printed documents is usually seen as solved problem freestyle handwritten word line still present a significant challenge. For example handwritten Arabic word lines are curvilinear. Linear or piecewise linear approximation is not accurate in general and neighboring handwritten Arabic word lines may be close or touch each other [3]. No well-defined baselines exist for most handwritten documents. It is still a significant challenge to segment Arabic word lines in handwritten documents. The word lines in a document must be split properly before recognition. The correctness and incorrectness of word line split directly affect accuracies of the word as well as character segmentation and consequently change the [4] accuracies of the word as well as character recognitions.

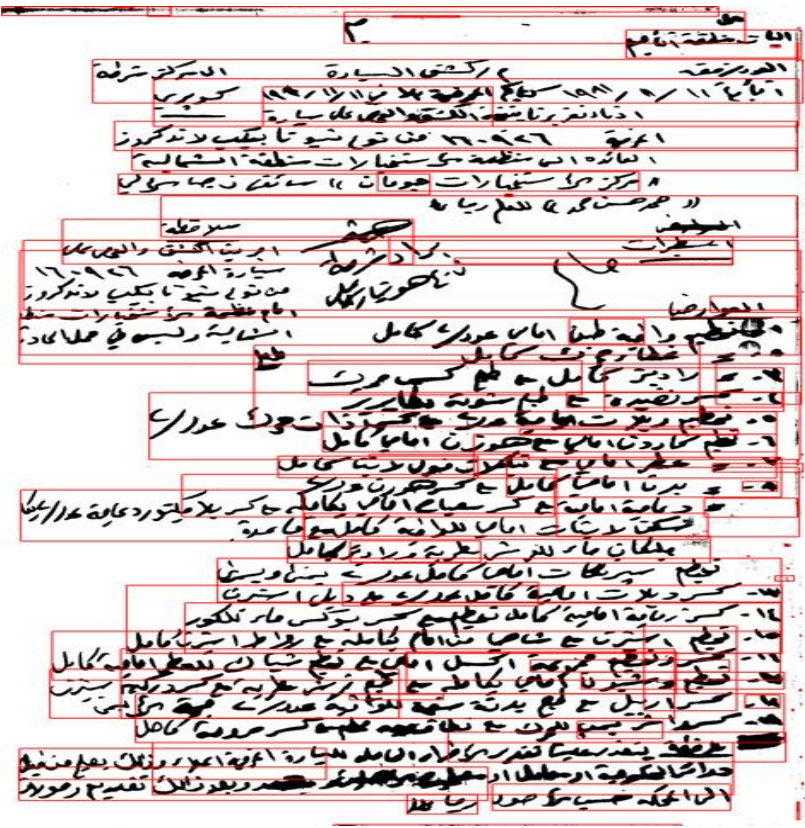

Fig 1: The Arabic word line segmentation using bounding boxes

It is not trivial to use bounding boxes to label overlapping curvilinear Arabic word lines, as shown in figure 1. A closed curve more suitable represents the boundary of an Arabic word line. Previous word line split prescript, such as connected component based prescript work directly on the input image. For connected component analysis, each pixel is treated equally and a transformation of one pixel may result in a dissimilar result. If two neighboring Arabic word lines touch each other through furthermore a single handwritten stroke, the segmentation algorithm fails. It's deal with this problem using a probabilistic approach. The delivery of black pixels in a document is not uniform. At the center of an Arabic word line, a pixel is more likely to be a black pixel than at Arabic word line emptiness.

Therefore, it evaluates a likelihood map, where each pixel represents the likelihood that it belongs to an Arabic word line. 
There are several techniques for word line segmentation is reported in the literature [5] but we are using the bounding box technique. They follow either the top-down approach or the bottom-up approach [6]. Using the bottom-up approach to segment the Arabic words starting from individual characters of the text. Bounding boxes are widely used in machine printed document analysis to represent the segmentation results of zones, Arabic word lines, and characters.

\section{THE SEGMENTATION}

The Segmentation is an important stage because the extent one can reach in partition of words, lines, or characters directly impresses the recognition rate of the Arabic script. Basically there are two types of segmentation firstly external segmentation, which is the segregation of various writing units, such as paragraphs, sentences, or words, and secondly internal segmentation, which is the segregation of letters, especially in cursively written words.

\subsection{External Segmentation}

It is the most baleful part of the document analysis, which is a needful step prior to the off-line character recognition Although document analysis is a relatively dissimilar research area with its own methodologies and techniques, split the document image into the text and non text regions is an integral part of the optical character recognition. The page layout analysis is accomplished in two stages: The first stage is the structural examine, which is concerned with the segmentation of the image into blocks of Arabic document components and the second stage one is the functional examine, which uses location, size, and various layout rules to label the functional Arabic content of document components [7].

\subsection{Internal Segmentation}

Albeit the methods have developed remarkably in the last decade and a variety of techniques have emerged, segmentation of cursive script into letters is still an undistinguished problem. The internal character segmentation strategies are divided into two types of Explicit Segmentation and Implicit Segmentation. In the Explicit Segmentation category, the segments are identified based on a character like properties. The process of cutting up the image into expressive components is given a special name dissection. The dissection is processes that examine an image without using a specific class of shape information. The criterion for good segmentation is the conciliation of general properties of the segments with those required for valid characters. In the Implicit Segmentation category, this segmentation strategy is based on recognition. It searches the image for components that coincide predefined classes. The segmentation is performed by the use of recognition confidence, including syntactic or semantic correctness of the exhaustive result [8].

\section{PREPROCESSING}

When Arabic data is scanned and digitized, the Arabic data may carry some unwanted noise. The consideration behind preprocessing is to bring out detail that is obscured and simply to highlight certain properties of interest in an image . Typical processing techniques are image smoothing using filters, contrast stretching, increasing dynamic range, image thresholding, histogram equalization etc. If the given Arabic document image is in color or grayscale pixel format, it is mutating to its black and white pixel format for further processing. These Arabic document images are noise free, skew free [9] and well formed and there are no broken and degraded characters. Using morphological transformations on to the goal image for removing the noise, line skew and to correct the broken and degraded character image. Hence, if the black pixel and white pixel Arabic document image has black to data pixels with white background pixels, as generally found, it is inverted to get white characters on black background. The Arabic images are also thinned to obtain the anatomical shape of the image that is just 1-pixel thick. The thinning algorithm used is a two iteration algorithm for binary regions with successive passes applied to contour points of an image using 8 nearness notations [10].

\section{PROPOSED CONNECTED COMPONENT LABELING AND BOUNDING BOX METHOD FOR ARABIC WORD}

In this paper we are proposing the bottom-up approach for Arabic word segmentation starting with the characters. From binary images analysis objects are usually extracted by means of the connected components labeling operation, which consists in assigning a masterly label to each maximal connected region of foreground Arabic word pixels. The connected components labeling scans an image and groups its pixels into components based on pixel connectivity all pixels in a connected component share similar pixel intensity value and are in some way connected with each other. Once all groups have been determined, each pixel is labeled with a gray level or a color (color labeling) according to the component it was assigned to. Extracting and labeling of various disjoint and connected components in an image is central to many automated image analysis applications. In the Arabic word cases the sub components of the character are nearby to each other and can be easily conjoin into a single component. The similar argument holds good for most of the other Languages [11]. Thus, to access particular characters, Using 8-connected component labeling. All Arabic words through connected components labeling are marked by the respective rectangular bounding boxes which just in close with them completely.

Let $\mathrm{F}$ indicate the input binary image pixel. A connected component analysis algorithm is applied to the foreground region of $\mathrm{F}$ to present the set of connected components. The each connected component, its respective bounding box the small rectangular box which restrains the component is calculated. A bounding box can depict by giving the coordinates of the upper left and the lower right corners of the box. The numbers of bounding boxes are eternally larger than the number of symbols since multiple bounding boxes are presented for multi component symbols. Arabic page segmentation scheme analyzes the spatial configuration of those bounding boxes of connected components to extract Arabic text lines, words and paragraphs.

The below figure shows the bounding boxes which enclose their connected components. The figure 2 shows Arabic 
document image and figure 3 shows the unfilled bounding rectangles (boxes) while figure 4 shows the same rectangles (boxes) filled with Black pixels. When the foreground Arabic objects of the binary image are white and the background black

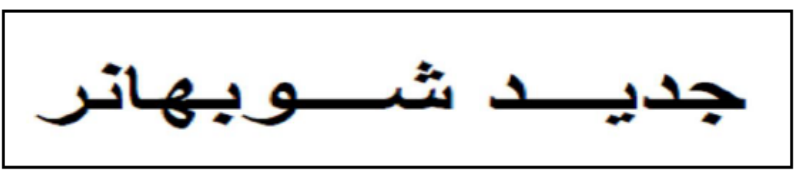

Fig 2 : The Arabic document image using bounding boxes

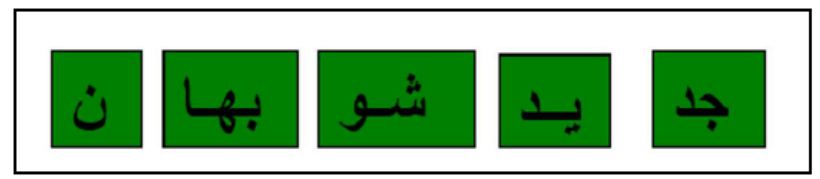

Fig 3 : The Arabic word unfilled bounding rectangles (boxes)

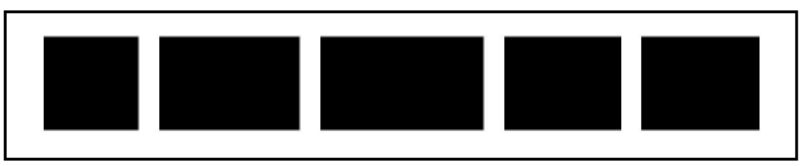

Fig 4 : The Arabic word rectangles (boxes) filled with Black pixels

The Arabic words Analysis of the spatial configuration of bounding boxes can be done by projecting those bounding boxes onto a straight line. The Arabic paper documents are usually written on the horizontal or vertical direction, projections of bounding boxes onto the vertical and horizontal lines are of distinctive interest [12]. Until projecting bounding boxes onto the horizontal or vertical line, they will collect onto that line, which results in the projection profile. A projection profile is a frequency delivery of the projected bounding box of the projection line. The bounding box projection profiles provide significant information about the number of bounding boxes aligned along the projection direction.

The algorithm groups the bounding boxes on each Arabic text line into bounding boxes of words. The algorithm first computes the projection profiles within each of the Arabic text line bounding boxes. When the foreground Arabic text objects of the binary image are white and the background black. In the Arabic text object is taken white (pixel value $=1$ ) while the background is black (pixel value $=0$ ). After that the algorithm considers each of the projection profiles as a onedimensional grayscale image, and thresholds each of the images with threshold value 1 to present an Arabic binary image. During the binarization, a symbol and or a broken symbol with various bounding boxes may be conjoined into one, as well as, those adjacent symbols within the same Arabic text line whose bounding boxes are overlapped with each other. But this will not reason any problem in the result of Arabic word extraction process, since appropriate algorithm extracts words by merging bounding boxes based on the lateral proximity of vicinal boxes. After similar binarization, the algorithm performs a morphological closing operation on each of the binarized Arabic text line projection profiles with structuring element of suitable shape. The length of the structuring element is resolute by analyzing the delivery of the run lengths of 0 on the binarized Arabic text line projection profile.

\section{THE F INTERVAL IN ARABIC WORD}

The horizontal spatial Arabic word separations between imminent bounding boxes are measured parallel to the $\mathrm{x}$-axis and hence called F Interval. These F Interval is the horizontal partition Interval between the corresponding imminent Arabic characters

and the remaining $\mathrm{F}$ Interval are the imminent Arabic word Interval of the document image. Arabic words Interval is the separation between the imminent words. The Arabic word Interval between two imminent words is the Interval between the last character of the first Arabic word and the first character of the second Arabic word. The Arabic word Interval is much wider than Arabic character Interval.

This paper's aim is to distinguish between the Arabic character Interval and word Interval and to fill the character Interval without filling the Arabic word Interval. The Arabic document image, the $\mathrm{F}$ Interval can have varying widths. These F Interval, represented by their widths form a set. We said the F Interval set. The F Interval set is a collection of all the distinct $\mathrm{F}$ Interval widths and Arabic character Interval and Arabic word Interval are mutually exclusive and exhaustive sub sets of the F Interval set. The Arabic character Interval has smaller widths than the Arabic word Interval. The average width of Arabic word Interval is much larger than that of the Arabic character Interval [13]. Hence from the $F$ Interval set, the character Interval set all letters and the Arabic word Interval set all words can be parted using a suitable threshold for separation.

\section{MORPHOLOGICAL DILATE IN ARABIC IMAGE}

The identification of objects within an Arabic image can be a very intricate task. One way to simplify the problem is to change the grayscale image into a binary image, in which each pixel is restricted to a value of either 0 or 1 . Mathematical morphology is a method of processing digital Arabic images based on shape [14]. The term morphology refers to the study of shapes and structures from a general scientific perspective. Also, it can be interpreted as shape study using mathematical set theory was done by Hadwiger [15]. In image processing, morphology is the name of a specific methodology for analyzing the geometric structure inherent within an Arabic image. The morphological filter, which can be constructed on the basis of the underlying morphological operations, is more suitable for shape analysis than the standard linear filters since the latter sometimes distort the underlying geometric form of the Arabic image.

The morphological operators transform the original Arabic image into another Arabic image through the interaction with the other Arabic image of a certain shape and size, which is known as the structuring element. Geometric features of the images that are similar in shape and size to the structuring element are preserved, while other features are suppressed [16]. Therefore, morphological operations can simplify the Arabic image data, preserving their shape characteristics and eliminate irrelevancies. In view of applications, 
morphological operations can be employed for many purposes, including edge detection, segmentation, enhancement of images and computer graphics.

The two principal morphological operations are dilation and erosion. In the dilation allows objects to expand, thus potentially filling in small holes and connecting disjoint objects. In the erosion shrinks objects by etching away (eroding) their boundaries. These operations can be customized for an application by the proper selection of the structuring element, which determines exactly how the objects will be dilated or eroded. The dilation process is performed by laying the structuring element $\mathrm{B}$ on the Arabic image A and sliding it across the Arabic image in a manner like to convolution. The difference is in the operation performed. It is best described in a sequence of steps. In firstly if

the origin of the structuring element coincides with a 'white' pixel in the Arabic image there is no move to change the next pixel. And secondly if the origin of the structuring element coincides with a 'black' in the image make black all pixels from the image covered by the structuring element.

In Morphological dilation $\mathrm{A}$ and $\mathrm{B}$ as sets in $\mathrm{Z}^{2}$, the dilation of A by B (usually A is an image and B is the structuring element), denoted by $A \oplus B$, is defined as

\section{$A A B=\left\{E Z^{2} \mid:=a+b\right.$, for somene $a$ a and $\left.b \in B\right\}$}

It can be shown that dilation is equivalent to a union of translation of the base Arabic image with respect to the structuring element:

$$
A \oplus B=\bigcup_{b \in B}(A)_{b}
$$

If the dilation is found by placing the center of the template over each of the foreground pixels of the base Arabic image and then taking the union of all the resulting copies of the structuring element, produced by using the translation. The dilation generally has an effect of expanding an image; so consequently small holes inside foreground can be filled. In another grasp, dilation can be a morphological operation on a binary image defined as

$$
A \oplus B=\left\{z \mid(\hat{B})_{z} \cap A \neq \phi\right\}
$$

This equation is based on obtaining the reflection of $\mathrm{B}$ about its origin and shifting this reflection by $\mathrm{z}$. The dilation of $\mathrm{A}$ by $\mathrm{B}$ is the set of all reshuffle, $\mathrm{z}$, such that B and A overlap by at least one element. Based on this interpretation, the equation above may be written as

$$
A \oplus B=\left\{z \mid\left[(\hat{B})_{z} \cap A\right] \subseteq A\right\}
$$

Although dilation is based on set operations, whereas convolution is based on arithmetic operations, the basic process flipping B about its origin and [17] successively reshuffling it so that it slides over set (Arabic image) $\mathrm{A}$ is analogous to the convolution process. Even though dilation of Arabic image A by structuring element B can be defined in several ways, all definitions have the same meaning and results in the same output. In this paper the proposed method the elaboration is restricted along the horizontal side only so that it impresses the F Interval but not the vertical separations. If the also horizontal dilation is made unidirectional (say from left to right) so that the appropriate width of dilation can be controlled. This is obtained by using a distinctive single row structuring element. The distinctive Structuring dilation element as described below. We select the structuring element as a single row matrix of $\mathrm{p}$ zeros followed by $(\mathrm{p}+1)$

$p=1,2,3,4,5 \ldots$ etc. Thus,

for $\mathrm{p}=1, \mathrm{ME} 1=\left[\begin{array}{lll}0 & 1 & 1\end{array}\right]$

for $\mathrm{p}=2, \mathrm{ME} 2=\left[\begin{array}{lllll}0 & 0 & 1 & 1 & 1\end{array}\right]$

for $\mathrm{p}=3, \mathrm{ME} 3=\left[\begin{array}{lllllll}0 & 0 & 0 & 1 & 1 & 1 & 1\end{array}\right]$

for $\mathrm{p}=3, \mathrm{ME} 4=\left[\begin{array}{lllllllll}0 & 0 & 0 & 0 & 1 & 1 & 1 & 1 & 1\end{array}\right]$

for $\mathrm{p}=3$, ME5 $=\left[\begin{array}{llllllllll}0 & 0 & 0 & 0 & 0 & 1 & 1 & 1 & 1 & 1\end{array}\right]$

In general for a detailed $\mathrm{p}$

MEp $=[0(p, 1) 1(p+1,1)](1)$

Where, $0(1, p)$ is a $1 x p$ matrix of 0 's and $1(p+1,1)$ is a $1 x(p+1)$ matrix of 1 's. The size of MEp itself is $1 x(2 p+1)$ and the special properties of MEp are if select only one row so that the dilation occurs only along the horizontal side another the total number of elements in it is odd and its center element is 1 with $p 0$ 's on its left and $p 1$ 's on its right so that the side of dilation is from left to right and the width of dilation is precisely p pixels. In this paper given bounding box Arabic document image dilation operation with MEp extends the right edges of the bounding boxes by $p$ pixels to right. The during dilation, if the right edge of a bounding box touches or traverses the left edge of its right vicinal then that $\mathrm{F}$ Interval is teeming up. Let $w$ be the width of an F Interval before dilation and $\mathrm{p}$ are the width of dilation then three conditions below.

If $\mathrm{p}<\mathrm{w}$, there is no merger after dilation. The $\mathrm{F}$ Interval is not teeming up. F Interval width is diminish from $\mathrm{w}$ to $(\mathrm{w}-\mathrm{p})$

If $\mathrm{p}==\mathrm{w}$, it results in a just merger. The $\mathrm{F}$ Interval is teeming up.

If $\mathrm{p}>\mathrm{w}$, it results in a merger with overlap. The $\mathrm{F}$ Interval is teeming up. 


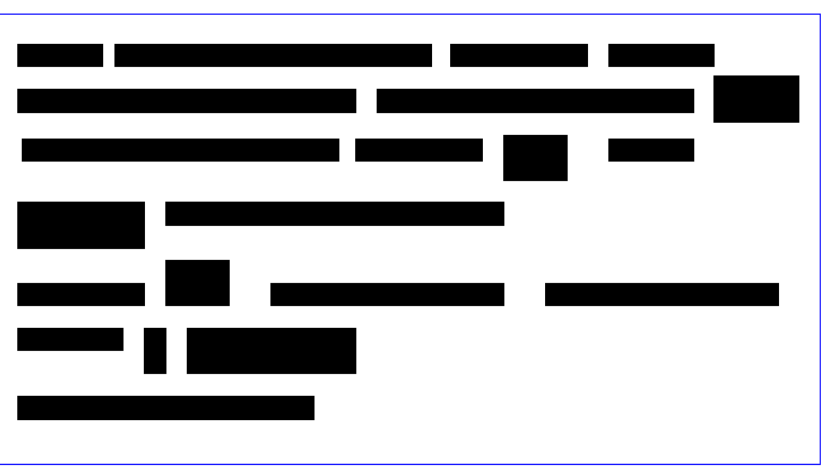

Fig 5: The Bounding Box 7 line Arabic Document Image before Dilation

In the bounding box image of an Arabic document commingle during dilation can be detected by counting number of connected components before and after dilation. When an $\mathrm{F}$ Interval is filled, commingle occurs and the number of separate components gets reduced by 1 .

All the remaining F Interval is obtained by the dilate operation which is successively encore with progressively increasing dilation width. The F Interval width list which contains ( $\mathrm{z} 1$, $\mathrm{z} 2, \mathrm{z} 3, \ldots)$ and the corresponding number of $\mathrm{F}$ Interval list containing ( pz1, pz2, pz3, ...) are determined. The pmax be the maximal $F$ Interval width of a given an Arabic document image. Once the dilation width $\mathrm{p}$ arrives $\mathrm{pmax}$ and all the $\mathrm{F}$ Interval of the

bounding box Arabic image are merged. The resulting Arabic image is a series of horizontal bars with no F Interval at all. An example where all $\mathrm{F}$ Interval is merged is shown in Figure 5 and Figure 6.

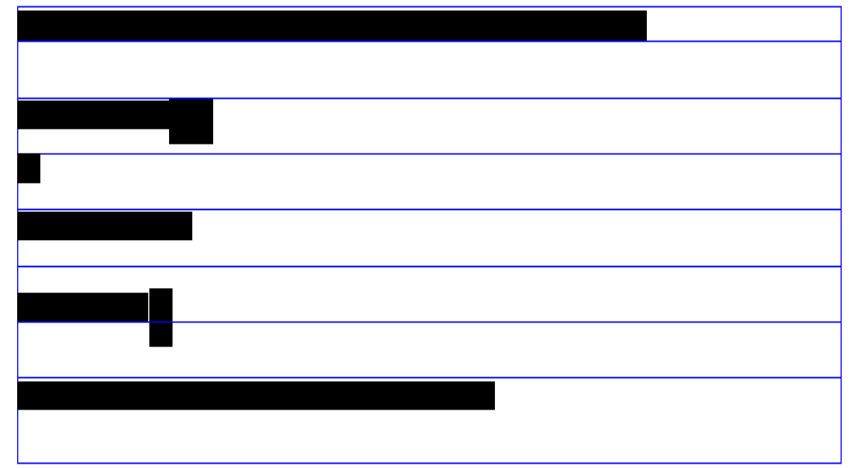

Fig 6 : The Bounding Box 7 line Arabic Document Image After Full F Dilation

\section{THE F INTERVAL ALGORITHEM IN ARABIC IMAGE}

The $\mathrm{P}$ is the binary filled bounding box Arabic Document image. Let the width of the image be Z. Let TL be the number of text lines.
Step1. The $\mathrm{Z}$ as the width of dilation, get the full $\mathrm{F}$ dilation of $\mathrm{P}$ to get $\mathrm{O}$. From $\mathrm{O}$, find the number of related components to get TL.

Step 2. The T1 the initial number of related components by applying bwlabel function to $\mathrm{P}$ Then that is obtained T1 as, [L $\mathrm{T} 1]$ = bwlabel $(\mathrm{P})$

Step 3. Initialize $F$ Interval _width and the number of $F$ Interval lists to null as, and $\mathrm{F}$ Interval width=[], and the number of F Interval =[]

Step 4. Start with $\mathrm{p}=1$ to set the dilation width to 1

Step 5. While T1>TL Then Construct MEp

Step 6. Do morphological dilation as, $\mathrm{O}=$ imdilate (P, MEp)

Step 7. IF T2, the number of related components after dilation as, Then [L T2] = bwlabel (O)

Step 8 . Then compare $\mathrm{T} 2$ to $\mathrm{T} 1$

If $(\mathrm{T} 1-\mathrm{T} 2)==0$,

Then Increment $\mathrm{p}$ by 1 as, $\mathrm{p}=\mathrm{p}+1$ and

GoTo step 5, Else

Step 9. A merger has occurred and the present dilation width $\mathrm{p}$ is one of the F Interval widths then

Step 10. Append this $\mathrm{n}$ into the $\mathrm{F}$ Interval _width list as, $\mathrm{F}$ Interval - width $=[\mathrm{F}$ Interval _width $\mathrm{p}]$ and append the number of $\mathrm{F}$ Interval list as, number of $\mathrm{F}$ Interval $=$ [number of $\mathrm{F}$ Interval (T1-T2)], Then

Step 11. Update $\mathrm{T} 1$ for the next iteration as, $\mathrm{T} 1=\mathrm{T} 2$ and Increment $\mathrm{n}$ by 1 as, $\mathrm{p}=\mathrm{p}+1$ and GoTo step 5

Step 12. End of while loop

\section{EXPRIMENTAL RESULTS OF BOUNDING BOX METHOED FOR ARABIC WORD}

In this paper number of $\mathrm{F}$ Interval list gives the number of $\mathrm{F}$ Interval who's In that behalf widths are present in the F Interval Arabic Word width list. The F Interval Arabic Word widths and the In that behalf number of F Interval for a typical Arabic document image are shown in Table 1. The graphical plot of the number of $F$ Interval versus the widths from the $F$ Interval Arabic word width list will show the F Interval Arabic word width delivery. The plot is shown in figure 7 and figure 8 . 
Table 1 : The F Interval Arabic Word Width

\begin{tabular}{|c|c|c|}
\hline $\begin{array}{c}\text { F Interval Arabic } \\
\text { word width }\end{array}$ & $\begin{array}{c}\text { Number of F Interval } \\
\text { Arabic word }\end{array}$ & $\begin{array}{c}\text { Arabic word Change } \\
\text { in F Interval width }\end{array}$ \\
\hline 3 & 174 & 2 \\
\hline 6 & 162 & 5 \\
\hline 9 & 157 & 3 \\
\hline 10 & 136 & 1 \\
\hline 13 & 101 & 1 \\
\hline 16 & 57 & 1 \\
\hline 18 & 21 & 4 \\
\hline 20 & 10 & 1 \\
\hline 23 & 7 & 1 \\
\hline 27 & 3 & \\
\hline & & \\
\hline
\end{tabular}

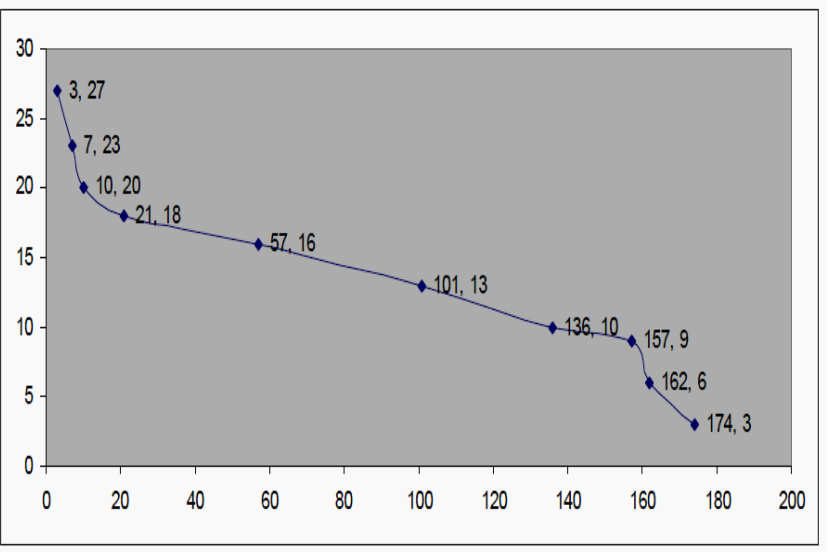

Fig 7 : The Distribution of F Interval Arabic Word Width and Number F Interval Arabic Word Plot

The letter mode and the word mode realm can be easily distinguished because of the clear separation between the two. The left to right dilation of the target image to this width will merge all the letter spaces while retaining word boundaries. The result is shown in figure 9 Arabic word segmentation blocks of the text and in figure 10 segmented Arabic word of the text. The F Interval Arabic word histogram has a zero value region between the upper Arabic letter Interval width and the lower Arabic word

Interval width. In this region the F Interval Arabic word width suddenly transformed from upper Arabic letter Interval width to lower Arabic word Interval width. It is determined by examining the F Interval Arabic word width list which gives the successive transformation in $\mathrm{F}$ Interval Arabic word width. The transformation is maximal at the transition from upper Arabic letter Interval width to lower Arabic word Interval width. You can behold column three of Table 1 in Arabic word Change in F Interval width.

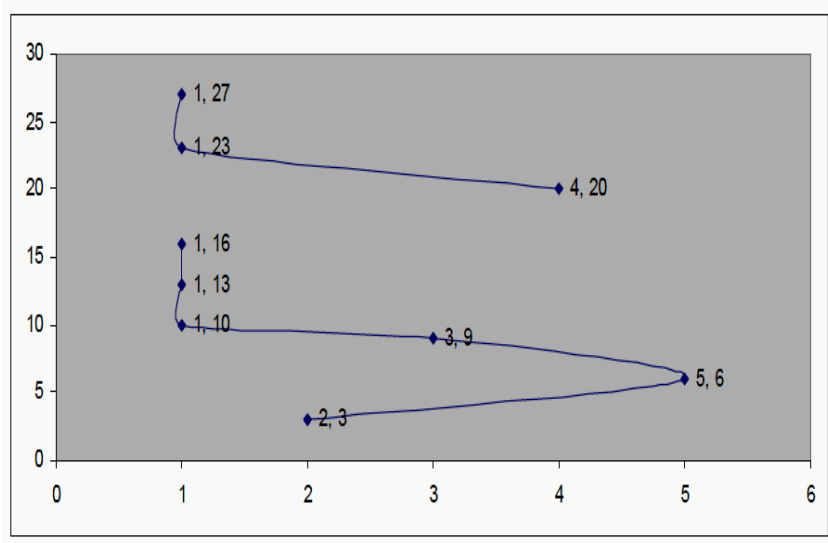

Fig 8 : The Distribution of F Interval Arabic Word Width and Arabic Word Change in F Interval Width Plot

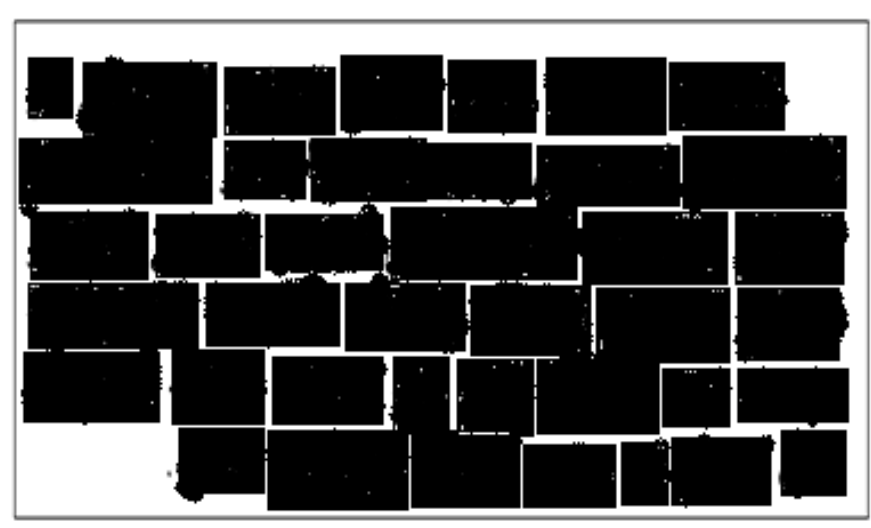

Fig 9 : The Arabic Word Segmentation Blocks of the Text

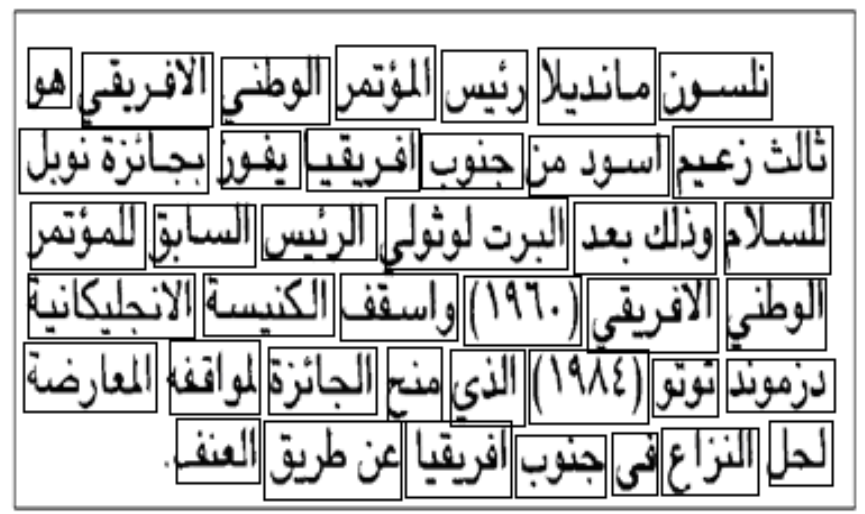

Fig 10 : The Segmented Arabic Word of the Text

\section{CONCLUSION}

The text line and word segmentation are one of the significant steps of optical character recognition systems. In optical character recognition the text lines in a document must be segmented deservedly before recognition. The text line and word segmentation are one of the baleful positions in machine recognition of any language. The accurate segmentation of individual symbols decides the accuracy of character recognition technique. It is used to slenderize image of a 
sequence of characters into sub images of individual epitome by segmenting lines and words. Arabic is the most popular language in the Middle East and North Africa. Hence proper segmentation of Arabic word is challenging. The Arabic text line and word segmentation in freestyle handwritten documents remains an open document analysis problem. The curvilinear Arabic text lines and small gaps between vicinal text lines present a challenge to algorithms developed for Arabic machine printed as well as Arabic hand printed documents. In this paper, we are proposed a novel and robust Arabic text line and word segmentation approach for freestyle handwritten documents using bounding box region. Its have instated consistently high precision on Arabic text line and word segmentation.

\section{REFERENCES}

[1]Likforman-Sulem, L., Zahour, A. and Taconet, B., "Text line Segmentation of Historical Documents: a Survey", International Journal on Document Analysis and Recognition, Springer, Vol. 9, Issue 2, pp.123-138, 2007.

[2]Wang Jin, Tang Bin-bin, Piao Chang-hao, Lei Gai-hui, "Statistical method-based evolvable character recognition system",IEEE International Symposium on Industrial Electronics (ISIE), pp. 804-808, July 2009

[3] Dr. Firoj Parwej, "An Empirical Evaluation of Off-line Arabic Handwriting And Printed Characters Recognition System", for published in the International Journal of Computer Science Issues (IJCSI), ISSN (Online): 16940814, which is published by SoftwareFirst Ltd, Doolar Lane, Mahebourg, Republic of Mauritius, vol. 9, Issue 6, pages 29 - 35, November 2012.

[4] Y. Li, Y. Zheng, and D. Doermann, "Detecting text lines in handwritten documents," in Proc. Int'l Conf. Pattern Recognition, 2006, pp. 1030-1033.

[5] S. Chen, R. M. Haralick, and I. Phillips, "Simultaneous word segmentation from document images using recursive morphological closing transform", Proceedings of the $3^{\text {rd }}$ ICDAR, pages 761-764, Aug. 1995.

[6] Jaekyu Ha \& Robert M. Haralick Ihsin, T. Phillips, "Document Page Decomposition by the Bounding-Box Projection Technique", IEEE Transactions on Systems, Man, and Cybernetics, Vol.18, No.1, pp.1118-1122, January 1995.

[7] Nafiz Arica , Fatos T. Yarman-Vural, "Correspondence An Overview of Character Recognition focused on OffLine Handwriting", IEEE Transactions on Systems, Man, and Cybernetics part C: Applications and Reviews, VOl.31, No.2, May 2001
[8] Richard G. Casey and Eric Lecolinet, "A Survey methods and Strategies in Character Segmentation", IEEE Transactions on Pattern Analysis and Machine Intelligence, Vol 18, No.7, July 1996

[9] Dr. Firoj Parwej , March 2013, " The State of the Art Recognize in Arabic Script through Combination of Online and Offline ",International Journal of Computer Science and Telecommunications (IJCST), published by Sysbase Solution (Ltd), UK, London (http://www.ijcst.org), Vol. 4, Issue 3, pp 43-48.

[10] E.L. Flores, “A Fast thinning algorithm", Proc. Of the SBT/IEEE International Telecommunications Symposium, vol.2, pp.594-599, Aug.1998.

[11] M. Dillencourt, H. Samet, M. Tamminen. , "A general approach to connected component labeling for arbitrary image representations", Journal of the ACM, 39(2):253280, April 1992.

[12] Jaekyu Ha \& Robert M. Haralick Ihsin, T. Phillips, "Document Page Decomposition by the Bounding-Box Projection Technique", IEEE Transactions on Systems, Man, and Cybernetics, Vol.18, No.1, pp.1118-1122, January 1995 .

[13] T. Pavlidis and J. Zhou, Page Segmentation and Classification, CVGIP, Graphical Models and Image Processing, Vol. 54, pp. 484-496, November 1992.

[14]S.J. Lee, R.M Haralick and L. G. Shapiro, "Morphologic edge detection," IEEE J. Robot. Automat., 3(2): 142-155, 1987.

[15] H. Hadwiger: "Vorlesungen über Inhalt, Oberfläche und Isoperimetrie", Springer Verlag, (1957)

[16] Luc Vincent, "Morphological grayscale reconstruction in image analysis: Applications and efficient algorithms," IEEE Trans. on Image Processing, 2(2):176-201, 1993.

[17] R. L. Haralick, S. R. Sternberg and X. Zhuang "Image Analysis Using Mathematical Morphology", IEEE Trans. Pattern Anal. Machine Intell., vol. 9, pp.523 -550 1987

Dr. Firoj Parwej Assistant Professor in the Department of Computer Science, Jazan University , Jazan , Kingdom of Saudi Arabia (KSA). He has authored a number of different journal and paper. His research interests include Soft Computing, Artificial Neural Network, Machine Learning, Pattern Matching \& Recognition, Artificial Intelligence, Image Processing, Fuzzy Logic, Bluetooth, Network and Database. He is a member of IEEE. 